\title{
Carbon dioxide rebreathing in non-invasive ventilation. Analysis of masks, expiratory ports and ventilatory modes
}

\author{
D. Samolski, N. Calaf, R. Güell, P. Casan, A. Antón
}

ABSTRACT: Carbon dioxide rebreathing in non-invasive ventilation. Analysis of masks, expiratory ports and ventilatory modes. D. Samolski, N. Calaf, R. Güell, P. Casan, A. Antón.

Background and Aim. Carbon dioxide $\left(\mathrm{CO}_{2}\right)$ rebreathing is a complication of non-invasive ventilation (NIV). Our objectives were to evaluate the ability of masks with exhaust vents $(\mathrm{EV})$ to avoid rebreathing while using positive pressure (PP) NIV with different levels of expiratory pressure (EPAP). Concerning volume-cycled NIV, we aimed to determine whether cylindrical spacers located in the circuit generate rebreathing.

Materials and methods. 5 healthy volunteers were eval- uated. Bi-level PP was used with 3 nasal and 2 facial masks with and without EV. Spacers of increasing volume attached to nasal hermetic masks were evaluated with volume NIV. Inspired $\mathrm{CO}_{2}$ fraction was analyzed.

Results. Rebreathing was zero with all nasal masks and EPAP levels. Using facial masks 1 volunteer showed rebreathing. There was no rebreathing while using all the spacers.

Conclusions. In healthy volunteers, nasal and facial masks with EV prevent rebreathing. In addition, the use of spacers did not generate this undesirable phenomenon. Monaldi Arch Chest Dis 2008; 69: 3, 114-118.

Keywords: $\mathrm{CO}_{2}$ rebreathing, Nasal and facial masks, Expiratory devices, Pressure and volume-cycled ventilators.

Respiratory Dept. Hospital de la Santa Creu i Sant Pau, Barcelona, Spain.

Supported in part by RTIC-ISCiii - Red Respira and ALAT-SEPAR grants.

Correspondence: Daniel Samolski M.D., Respiratory Dept., Hospital de la Santa Creu i Sant Pau, Sant Antoni M ${ }^{a}$ Claret 167, 08025 Barcelona, Spain; e-mail: dsamolski@gmail.com

\section{Introduction}

Portable ventilators used in non-invasive ventilation (NIV) may provoke carbon dioxide $\left(\mathrm{CO}_{2}\right)$ rebreathing due to the use of a single-limb circuit [1]. The mode of ventilation, the type of mask, the expiratory device and the expiratory pressure level setting may also play a key role in this event [2-6].

Several masks currently used in positive pressure ventilation have exhaust vents (EV) that function as an expiratory port. In addition, some authors $[2,7]$ have shown that specific anti-rebreathing expiratory devices (ARD) minimise or prevent rebreathing when used in conjunction with hermetic masks. To our knowledge, no studies have been conducted to determine whether the newly designed EV equipped masks act in a similar way when used in combination with portable pressurecycled ventilators.

While using volume-cycled ventilation, exhalation is allowed by a pneumatic valve that, if positioned next to the mask, is uncomfortable because of its size and form. It is therefore necessary to add a flexible cylindrical spacer of variable size to separate the mask from the valve. Such a structure may interfere with the expiratory phase and generate a dead space that could potentially cause rebreathing. No studies have evaluated this issue.

The aim of the present study was to assess the ability of EV equipped nasal and facial masks to prevent rebreathing while using a portable pressure-cycled ventilator, and to quantify the minimum EPAP level needed to do this. Using volumecycled ventilation, we also aimed to evaluate the maximal volume of a cylindrical spacer still effective in preventing rebreathing.

\section{Materials and methods}

The study was conducted in the pulmonary function laboratory of our hospital. All volunteers provided written consent prior to participate in this study. Moreover, the project was approved by the Hospital Ethics Committee.

\section{Protocol}

Five healthy volunteers were evaluated. None of them had a prior history of smoking or respiratory diseases or were using drugs at the time of the study.

- Pressure-cycled ventilation: a VPAP III ventilator (ResMed, North Ryde, Australia) with 
its own single-limb circuit was used for the study. Three different nasal masks and two facial masks were evaluated. Two nasal masks had an EV (UltraMirage and Mirage, ResMed, North Ryde, Australia). The one out of EV (UltraMirage, ResMed, North Ryde, Australia) was connected to an ARD (Plateau valve, Respironics, Murrysille, PA, USA). Fig. 1. One facial mask (UltraMirage, ResMed, North Ryde, Australia) was EV equipped, and the other (Mirage, ResMed, North Ryde, Australia), without EV, was connected to the ARD. Using sealed envelopes each containing the specification for a single mask and pressure level combination among those to be tested, volunteers were randomly crossed over to receive NIV sessions with the type of mask and pressure selected. We set a fixed inspiratory pressure (IPAP) of $14 \mathrm{cmH}_{2} \mathrm{O}$ and an increasing expiratory pressure (EPAP) of 4, 6, 8 and $10 \mathrm{cmH}_{2} \mathrm{O}$.

- Volume-cycled ventilation: we used a Breas PV 501 ventilator (Breas Medical, Swedish) with its own single-limb circuit and expiratory valve. An hermetic nasal mask was employed (UltraMirage without EV, ResMed, North Ryde, Australia).

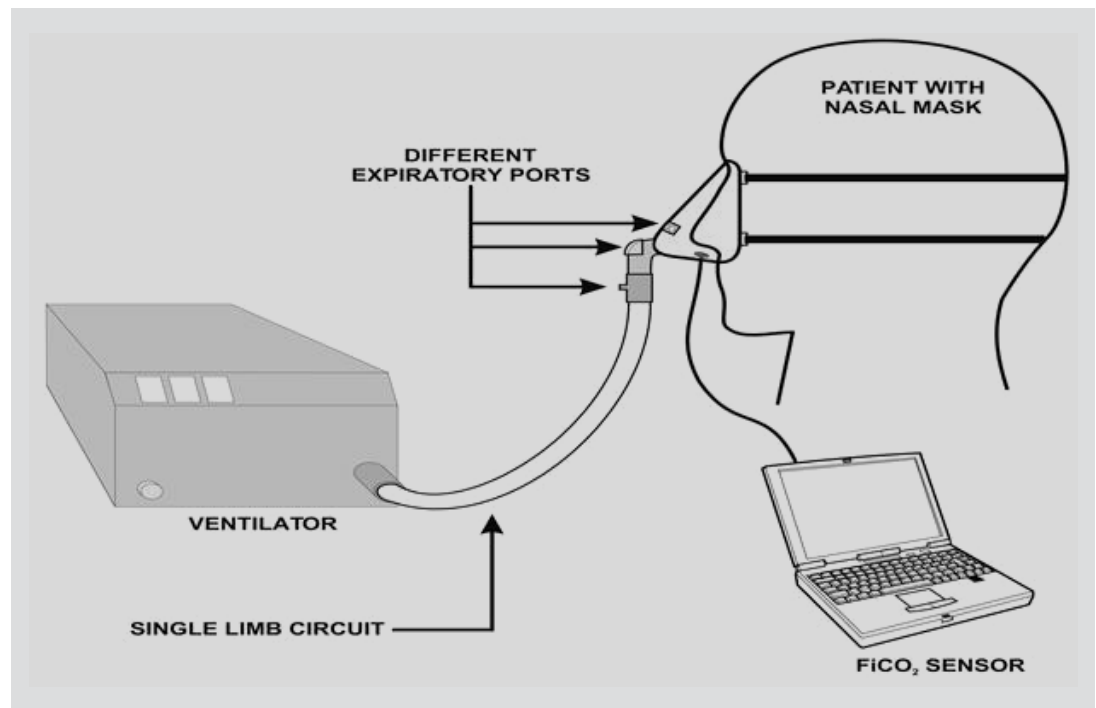

Fig. 1. - Pressure-cycled ventilator and nasal mask with different expiratory ports.

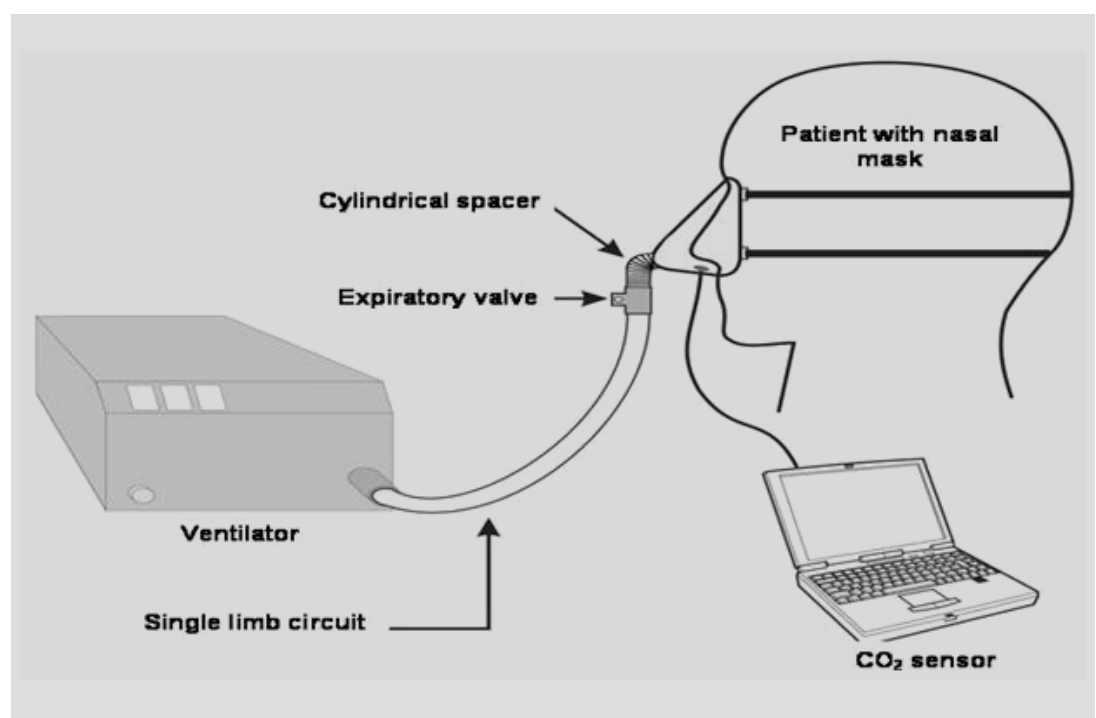

Fig. 2. - Volume-cycled ventilator and cylindrical spacer between the mask and the expiratory valve.
The cylindrical spacers located between the mask and the expiratory valve had three different volumes $(43,85$ and $176 \mathrm{ml}$.) that were selected in random order using sealed envelopes each containing the specification for a volume to be tested. All volunteers underwent NIV sessions receiving a tidal volume of $8-10 \mathrm{ml} / \mathrm{kg}$, a respiratory rate of 16-18 cycles/minute and an I/E relation of 1:2. Fig. 2.

The inspired $\mathrm{CO}_{2}$ fraction $\left(\mathrm{FiCO}_{2}\right)$ was continuously analysed using a thin sampling tube located in the mask, close to the nostrils. This tube was connected to a paramagnetic $\mathrm{CO}_{2}$ analyser (NDIR) with a $0-10 \%$ response lower than 130 milliseconds and a precision of $+/-0.05 \%$ (Medical graphics system, St. Paul, Minnesota, USA). Fig. 3. The paramagnetic $\mathrm{CO}_{2}$ analyser was calibrated using atmospheric air and a calibration gas mixture of $\mathrm{CO}_{2}(6.87 \%), \mathrm{O}_{2}(50.3 \%)$ and $\mathrm{N}_{2}(42.83 \%)$ (Abello Linde SA, Barcelona, Spain). The gain and the zero levels were checked prior to each change in the pressure level, the type of mask or the spacer's volume. Moreover, we performed the calibration before carrying out the study in each volunteer.

Volunteers were seated in a semi-recumbent position and each mode of ventilation was evaluated for five minutes. They were advised to keep their mouths closed while using nasal masks to avoid leaks through it. A wash-out period consisting of 15 minutes of room air breathing was performed between each NIV session.

\section{Data analysis}

The $\mathrm{FiCO}_{2}$ level was measured in 15 respiratory cycles after adaptation to each level of ventilation. The average $\mathrm{FiCO}_{2}$ level from these breaths was then considered for further analysis. According to the study design, differences of the mean $\mathrm{FiCO}_{2}$ level in all the ventilatory conditions tested were planned to be evaluated with a Friedman 2 way ANOVA non parametric test since low $\mathrm{FiCO}_{2}$ values, but different from zero, were expected before the study completion. Differences will be considered statistically significant when $p<0.05$.

\section{Results}

Ventilation was well tolerated in all volunteers regardless of the mode of ventilation, the interface or the volume of the spacers.

With reference to pressurecycled ventilators, as shown in Fig. 4, $\mathrm{FiCO}_{2}$ was zero while 


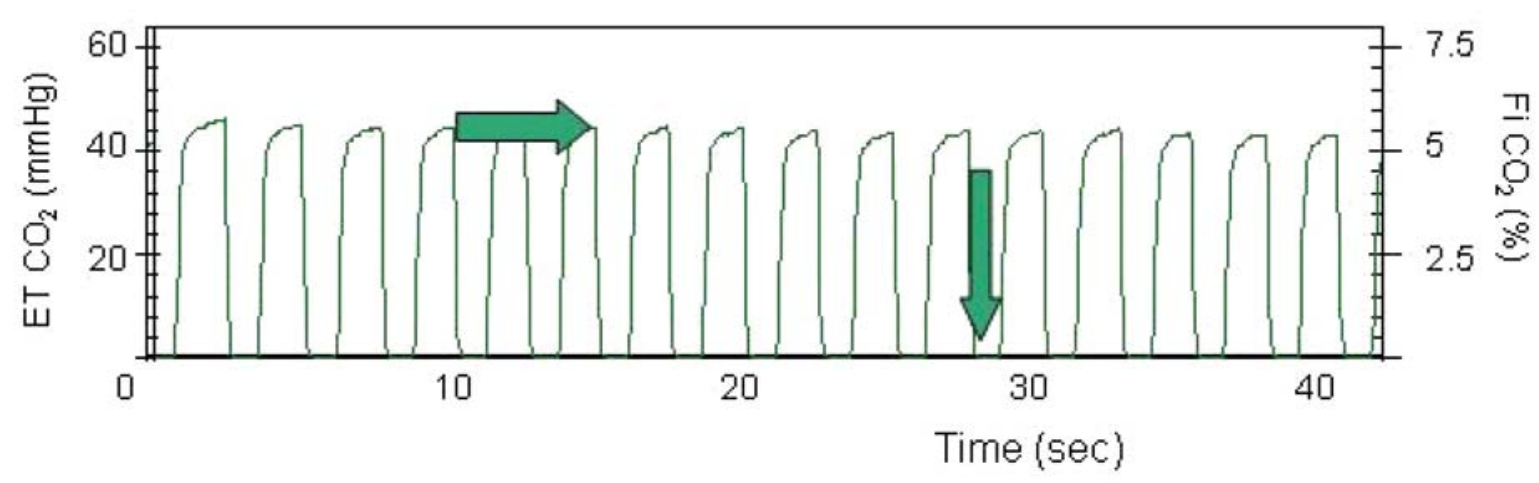

Fig. 3. - Continuous analysis of $\mathrm{CO}_{2}$ inside the mask.

Horizontal arrow: end tidal $\mathrm{CO}_{2}\left(\mathrm{ETCO}_{2} \mathrm{mmHg}\right)$. Vertical arrow: inspired $\mathrm{CO}_{2}$ fraction $\left(\mathrm{FiCO}_{2} \%\right)$.

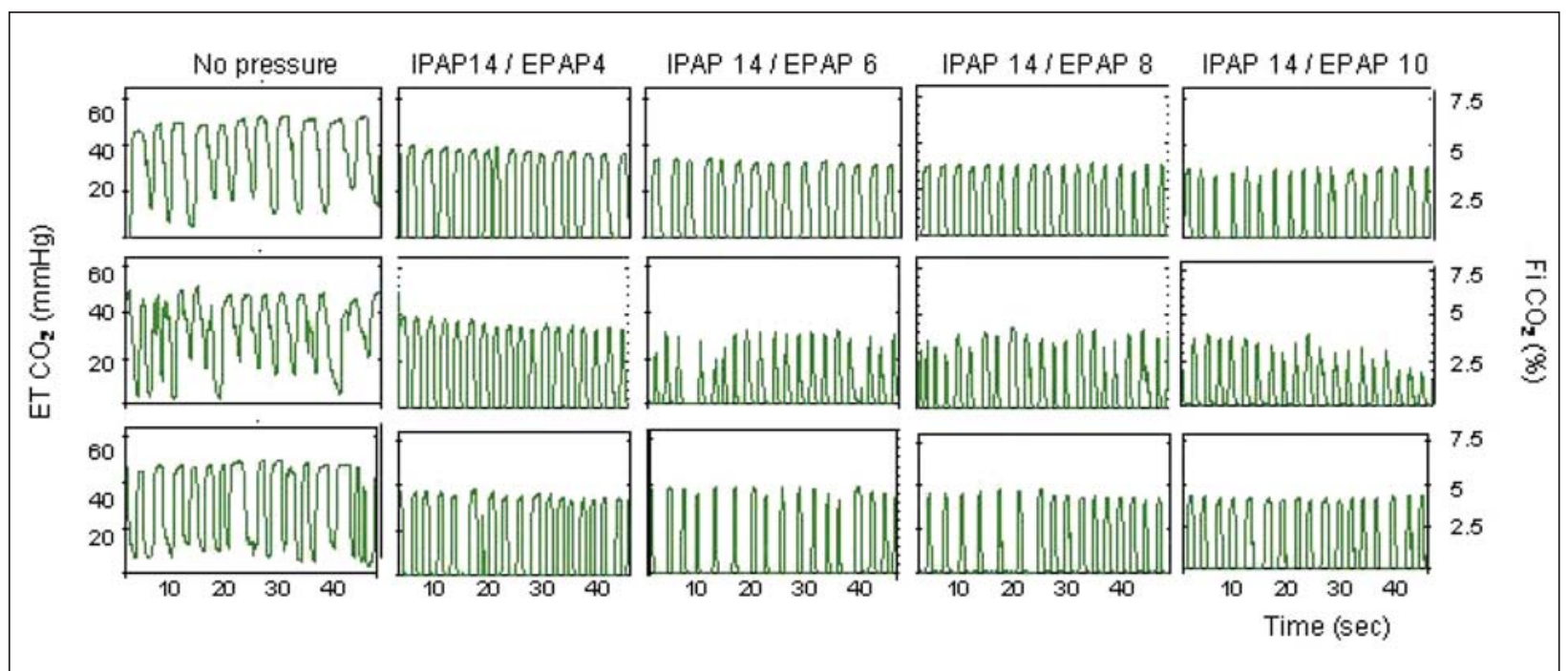

Fig. 4. - Inspired $\mathrm{CO}_{2}$ fraction $\left(\mathrm{FiCO}_{2}\right)$ during nasal mask ventilatory assistance with increasing levels of EPAP.

Top: nasal mask (UltraMirage, ResMed, Australia) with exhaust vent (EV). Middle: nasal mask (Mirage, ResMed, Australia) with EV. Bottom: Hermetic nasal mask (UltraMirage without EV, ResMed, Australia) joined to an anti-rebreathing expiratory port (Respironics, USA). Data obtained from volunteer \#5.

using all the nasal masks tested and their corresponding expiration ports. An EPAP as low as 4 $\mathrm{cmH}_{2} \mathrm{O}$ was useful to prevent rebreathing in all the conditions tested.

As Fig. 5 shows, $\mathrm{FiCO}_{2}$ was zero while using the EV equipped facial mask, whatever the EPAP used. The hermetic mask connected to an ARD showed no rebreathing in all except one volunteer. In this individual, the $\mathrm{FiCO}_{2}$ was $0.2 \%$ and it was not modified with the increase in EPAP.

While using volume-cycled ventilators, rebreathing was zero for all the volumes of spacers studied, as seen in Fig. 6.

\section{Discussion}

This study shows that newly designed nasal and facial masks with EV prevent $\mathrm{CO}_{2}$ rebreathing, even with an EPAP as low as $4 \mathrm{cmH}_{2} \mathrm{O}$ in normal subjects. Likewise, a cylindrical spacer located between the mask and the expiratory valve did not generate rebreathing during volume ventilation.
The phenomenon of rebreathing is generally considered a potential cause of failure of NIV treatment [1]. Since the first description by Ferguson et al [2] in 1995, little has been published on this subject. It is well known that the use of a single-limb circuit is its basic causal mechanism and that it can be modified by the mode of ventilation $[2,3]$ and the type of mask or expiratory port $[4,5$, 9-11]. The use of positive pressure during the expiratory phase could help with the "wash out of $\mathrm{CO}_{2}$ " through the holes of the mask. As a matter of fact, an appropriate EPAP level has been claimed to be critical in preventing rebreathing [2, 11].

Ferguson et al [2], using a specific ARD, found that an EPAP level of 6-8 $\mathrm{cmH}_{2} \mathrm{O}$ was necessary to prevent rebreathing. In our work, using a mask with EV, we were able to reduce the EPAP to $4 \mathrm{cmH}_{2} \mathrm{O}$ without rebreathing. Our results are in agreement with those described by Schettino et al [5] who also used masks with EV. This improvement in preventing $\mathrm{CO}_{2}$ rebreathing could be related to improve $\mathrm{CO}_{2}$ kinetics with this type of masks with EV [4]. Another important aspect to 


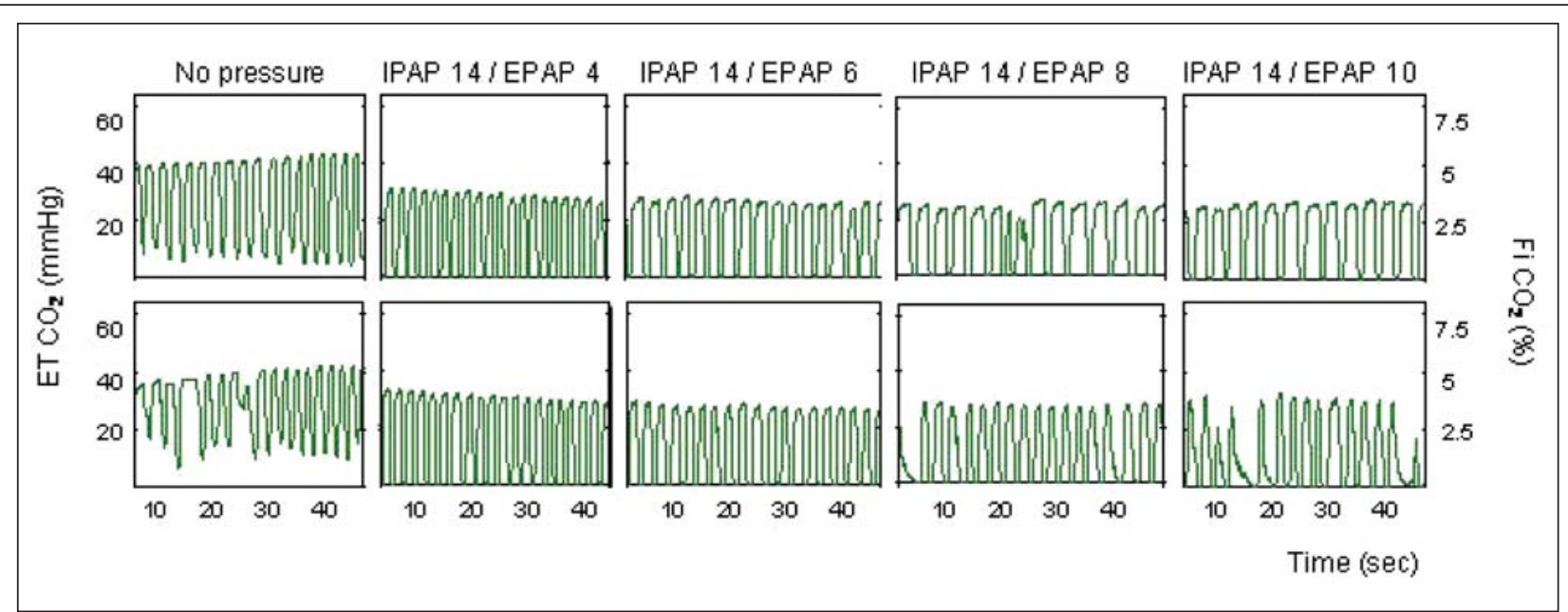

Fig. 5. - Inspired $\mathrm{CO}_{2}$ fraction $\left(\mathrm{FiCO}_{2}\right)$ during facial mask ventilatory assistance with increasing levels of EPAP.

Top: Facial mask with EV (UltraMirage, ResMed, Australia). Bottom: Hermetic facial mask (Mirage, ResMed, Australia) joined to an antirebreathing expiratory port.

Data obtained from volunteer \#3.

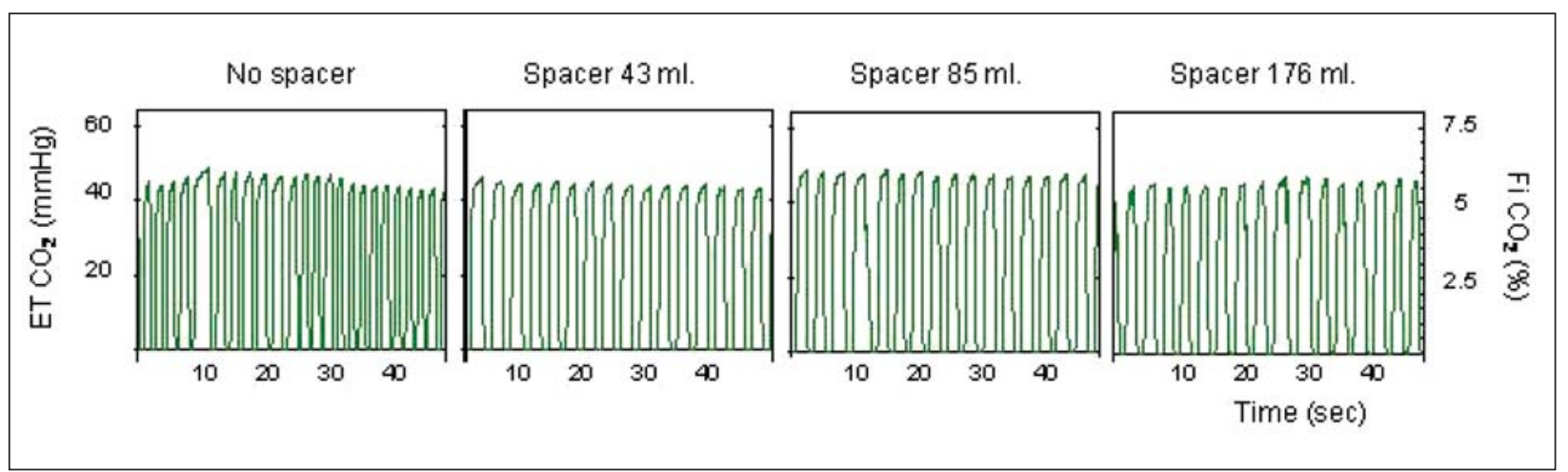

Fig. 6. - Inspired $\mathrm{CO}_{2}$ fraction $\left(\mathrm{FiCO}_{2}\right)$ inside the mask while using flexible cylindrical spacers of increasing volume.

Data obtained from volunteer \#2.

take into consideration is that the expiratory devices could worsen expiratory resistance. This situation was well described by Lofaso et al [3] who suggested that this type of expiratory plateau port could increase the positive end-expiratory pressure (PEEP) due to its higher expiratory resistance. Hence, the increase in expiratory resistance should be taken into account when using ARDs.

Schettino et al [5] evaluated the effects of mask volume in rebreathing. These authors reported, as was expected, that the volume of the mask was associated with $\mathrm{CO}_{2}$ rebreathing. Saatci et al [8] found that dead space is higher with the facial mask but that it could be partially offset using appropriate levels of positive pressure or adding an EV to enhance mask ventilation. In our work, only one volunteer showed an increase in $\mathrm{FiCO}_{2}$ while using a hermetic facial mask connected to an ARD. This phenomenon was not modified even when the EPAP level was increased. On the other hand, we did not observe rebreathing with the facial mask with EV, even with EPAP level as low as $4 \mathrm{cmH}_{2} \mathrm{O}$.

The addition of a spacer with an internal volume up to $176 \mathrm{ml}$, acting as dead volume, did not generate rebreathing. This finding suggests that pneumatic valves are effective in preventing re- breathing. On the other hand, we cannot rule out the possibility that a greater volume of the spacers could generate rebreathing. However, with a spacer as large as $176 \mathrm{ml}$. (the highest volume in our study), the mask can be comfortably separate from the expiratory valve. Thus, longer spacers with higher internal volume seem unnecessary. To our knowledge, this is the first study addressing this issue.

Reports in literature about the clinical significance of rebreathing are few and contradictory. Comparing an ARD with a standard valve, Hill et al [7] reported no clinical or gasometric differences in patients undergoing chronic NIV. By contrast, Farré et al [6] suggested that rebreathing could be so deleterious to lead to CPAP failure, an event that could be prevented by using an anti-rebreathing valve.

Our study in healthy volunteers show that the masks tested had little or no $\mathrm{CO}_{2}$ rebreathing. However, the "dead space effect" of these masks may negatively affect already hypercapnic patients who show a rapid and shallow breathing. For this reason, further studies on this kind of patients are warranted to assess the $\mathrm{CO}_{2}$ wash-out effectiveness of newly designed masks during assisted ventilation. 


\section{Technical remarks and limitations of the study}

Rebreathing can be detected by measuring $\mathrm{CO}_{2}$ inside the mask during the ventilatory cycle. It is not clear how or at what moment $\mathrm{CO}_{2}$ should be measured to quantify rebreathing. Some authors suggest that end-tidal of $\mathrm{CO}_{2}\left(\mathrm{ETCO}_{2}\right)$ is the best parameter to evaluate this phenomenon $[5,7$, 11]. Others consider that $\mathrm{FiCO}_{2}$ is better than ET$\mathrm{CO}_{2}$ measurement [2, 9]. In our study, we analysed the $\mathrm{CO}_{2}$ present inside the mask at the beginning of the inspiration (proto-inspiration) because, in our opinion, it better reflects the amount of $\mathrm{CO}_{2}$ rebreathing, as this is the real quantity of this gas that the patient will inspire. Likewise, it is logical to suppose that a greater $\mathrm{ETCO}_{2}$ could generate rebreathing. However, this depends not only on the amount of expired $\mathrm{CO}_{2}$ but also on the ability of the expiratory port to eliminate it. In view of these considerations, we took into account the $\mathrm{FiCO}_{2}$ and not the $\mathrm{ETCO}_{2}$ to evaluate the rebreathing.

Secondly, we analysed only a few of the masks that are currently available to make the study feasible. Moreover, it could be supposed that other similarly designed interfaces would yield similar results.

Thirdly, an open ventilatory system, like the one used in this work, did not allow us to quantify exactly the ventilatory mask flows; consequently, the $\mathrm{CO}_{2}$ rebreathing was showed as a percentage and not as a real amount.

Fourthly, we do not use any dyspnoea or tolerance scales to assess objectively mask comfort. We deduced that ventilation was well tolerated in all volunteers from the fact that all of them could fulfill and complete the whole study without reporting significant troubles or complaints with each mask or expiratory port, the mode of ventilation and the spacer.

Finally, with reference to statistical analysis, the absolute values obtained were not adequate for applying the planned statistical analysis. $\mathrm{FiCO}_{2}$ amounting almost invariably to zero. However, the repetition of this result strongly supports our conclusions. Thus, we decided to show only raw data with no more analysis.

In conclusion, we show that in healthy volunteers $\mathrm{CO}_{2}$ rebreathing is not a common issue in newly designed masks with incorporated $\mathrm{EV}$ even when EPAP is low or when cylindrical spacers are added to the circuit during volume-cycled ventilation. Our results should be contrasted in future with a clinical trial in hypercapnic patients.

Acknowledgements: The authors thank Carolyn Newey and Daniela Bordet for their assistance in editing the manuscript. Also they thank Marina Khoury for her assistance in data analysis.

\section{References}

1. Hill NS. Problems, remedies, and strategies to optimize the success of noninvasive ventilation. In: Hill NS, editor. Non invasive positive pressure ventilation. Armonk NY: Ed. Futura publishing company 2001: 187213.

2. Ferguson GT, Gilmartin $\mathrm{M} . \mathrm{CO}_{2}$ rebreathing during BiPAP ventilatory assistance. Am J Respir Crit Care Med 1995; 151: 1126-1135.

3. Lofaso F, Brochard L, Touchard D, Hang T, Harf A, Isabey D. Evaluation of carbon dioxide rebreathing during pressure support ventilation with airway management system (BiPAP) devices. Chest 1995; 108:772778 .

4. Zhang X ChR, He G. Modification of facial mask on the dead space effect in non invasive mask ventilation. Zhonghua Jie He He Hu Xi Za Zhi 2000; 23: 734-736.

5. Schettino GP, Chatmongkolchart S, Hess DR, Kacmarek R. Position of exalation port and mask design affect $\mathrm{CO}_{2}$ rebreathing during non-invasive positive pressure ventilation. Crit Care Med 2003; 31: 2178-82.

6. Farre R, Montserrat JM, Ballester E, Navajas D. Potential rebreathing after CPAP failure during sleep. Chest 2002; 121: 196-200.

7. Hill NS, Carlisle C, Kramer N. Effect of a nonrebreathing exhalation valve on long term nasal ventilation using a bilevel device. Chest 2002; 122: 84-91.

8. Saatci E, Miller DM, Stell IM,Lee KC, Moxham J. Dynamic dead space in face masks used with non-invasive ventilators: a lung model study. Eur Respir J 2004; 23: 129-135.

9. Taccone P, Hess D, Caironi P, Bigatello LM. Continuous positive airway pressure delivered with a helmet. Effects on carbon dioxide rebreathing. Crit Care Med 2004; 32: 2090-2096.

10. Racca F, Appendini L, Gregoretti C. Effectiveness of mask and helmet interfaces to deliver noninvasive ventilation in human model of resistive breathing. $J$ Appl Physiol 2005; 99: 1262-1271.

11. Mediano O, Garcia Rio F, Villasante C. Comparacion de la reinhalación de anhídrido carbónico originada por 3 mascarillas durante la aplicacion de CPAP. Arch Bronconeumol 2006; 42: 189-93. 